\title{
The emerging role of the Ikaros stem cell factor in the neuroendocrine system
}

\author{
Shereen Ezzat ${ }^{1}$ and Sylvia L Asa ${ }^{2}$ \\ ${ }^{1}$ Department of Medicine and ${ }^{2}$ Department of Pathobiology, Ontario Cancer Institute, Princess Margaret Hospital, University Health Network, Toronto, Ontario, \\ Canada M5G-1X5 \\ (Correspondence should be addressed to S Ezzat who is now at Ontario Cancer Institute, 610 University Avenue, \#8-327, Toronto, Ontario, Canada M5G-2M9; \\ Email: shereen.ezzat@utoronto.ca)
}

\begin{abstract}
In this review, we cover the evidence implicating lkaros as a key factor whose transcriptional actions and chromatin remodeling properties determine the fate of hypothalamic neuroendocrine and pituitary cell population expansion. We propose that the governing mechanisms involved in the regulation and action of lkaros are of importance during developmental as well as neoplastic transitions.
\end{abstract}

Journal of Molecular Endocrinology (2008) 41, 45-51

\section{Introduction}

The process of anterior pituitary cell development and differentiation follows a highly specific pattern and temporal sequence. Several putative transcriptionregulating proteins have been identified in the pituitary and have been implicated as key elements in the definition of cell-specific phenotypes and the regulation of hormone gene expression (Asa \& Ezzat 1998, 1999, 2002). Which of the lineage-regulating factors predominates in determining the lineage choice and expansion can be decided not only by the expression and relative concentration of the transcription factor but also by the chromatin accessibility at their cognate sites.

Ikaros was originally described as a transcription factor that binds to regulatory sequences of genes expressed in lymphoid cells (Molnar et al. 1996, Georgopoulos et al. 1997). The single copy gene contains seven exons that can, by alternative splicing, give rise to eight isoforms (Fig. 1) (Hahm et al. 1994, Molnar \& Georgopoulos 1994, Sun et al. 1996). All Ikaros isoforms share a common C-terminal domain that contains a transcription activation motif and two zinc finger motifs required for hetero- and homodimerization among the $\mathrm{Ik}$ isoforms and for interactions with other proteins (Winandy et al. 1995, Sun et al. 1996, Hahm et al. 1998). The N-terminal region includes a domain with zinc finger motifs critical for DNA binding. Ikaros isoforms differ in the number of N-terminal DNA-binding zinc fingers that differentiate them into members with or without DNAbinding properties (Hahm et al. 1994, Molnar \& Georgopoulos 1994). Only Ik1-3 contain the requisite three or more amino $(\mathrm{N})$-terminal zinc fingers that confer high-affinity binding to an Ikaros-specific core DNA sequence motif in the promoters of target genes (Sun et al. 1996). Dominant-negative (dn) forms of Ikaros lack the DNA-binding domain.

The various isoforms can act either as transcriptional activators or repressors as part of an integral component of a functionally diverse chromatin remodeling network (Molnar et al. 1996). It has become readily evident that the Ikaros system represents an example of a finely tuned system with distinct regulatory functions, particularly during early developmental stages in restricted target tissues.

In the earliest studies, Ikaros expression was identified in the brain and pituitary (Georgopoulos et al. 1992), but this was largely left uninvestigated. Our interest in this gene was precipitated by our identification of abundant expression of Ikaros in the anterior pituitary gland and hypothalamic neurons where we have demonstrated important functions for this factor in the regulation of multiple hormones including proopiomelanocortin (POMC), growth hormone (GH), prolactin (PRL), and GH-releasing hormone (GHRH). We have also identified a role for Ikaros in modulating neuroendocrine cell growth and survival functions (Ezzat et al. 2003, 2006b).

DOI: 10.1677/JME-08-0045 Online version via http://www.endocrinology-journals.org 


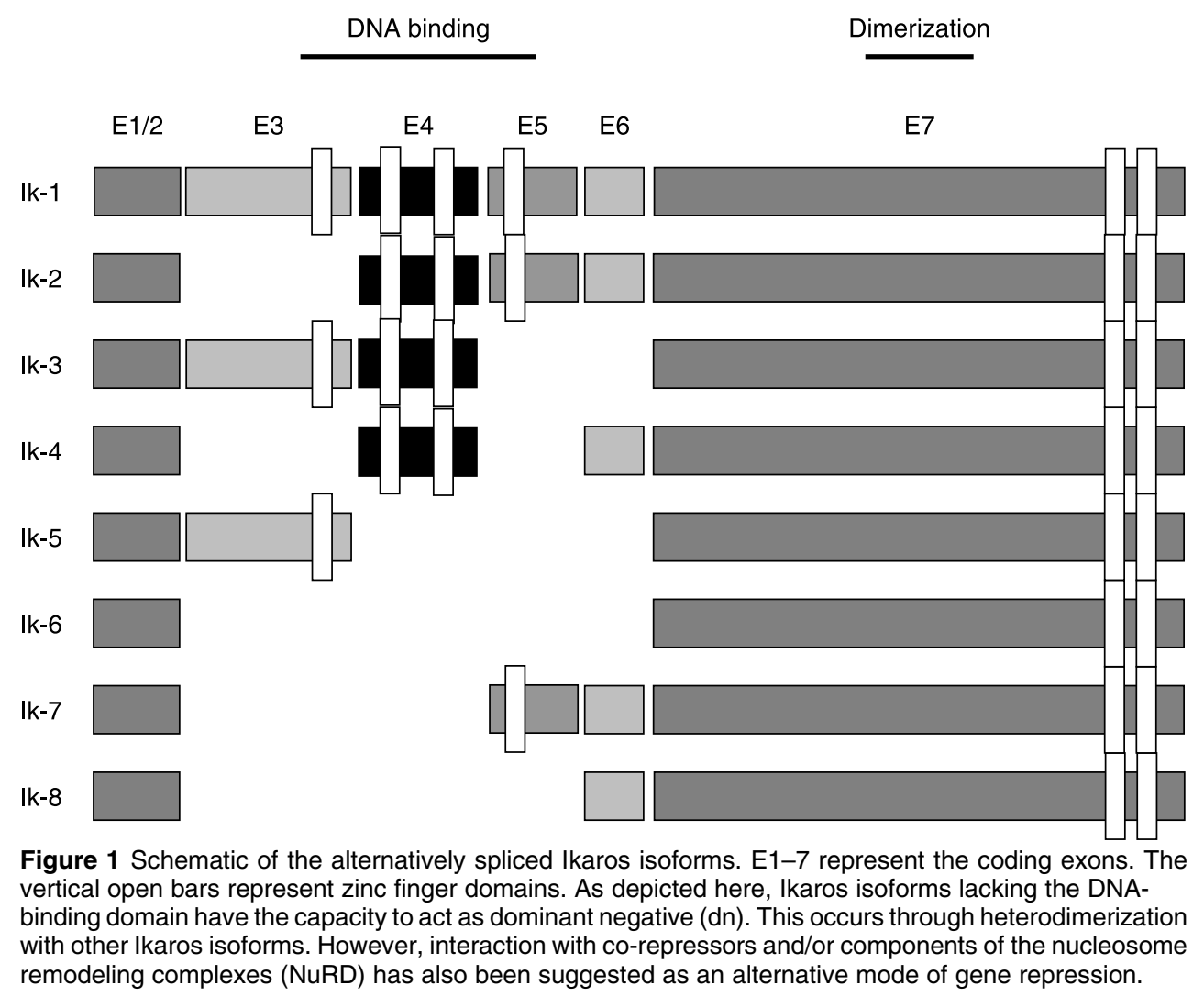

In this review, we cover the evidence implicating Ikaros as a key factor whose transcriptional actions and chromatin-remodeling properties direct hypothalamic neuroendocrine and pituitary cell population expansion during the development. We propose that the governing mechanisms involved in the regulation and action of Ikaros are of importance during developmental as well as neoplastic transitions.

\section{The importance of Ikaros in hematopoietic stem cell commitment}

Gene-targeting experiments have firmly established that nuclear factors encoded by the Ikaros gene are essential for normal lymphoid development (Cortes et al. 1999). Mice homozygous for a null mutation in Ikaros display deficient B- and T-cell differentiation (Wang et al. 1996, Winandy et al. 1999), whereas those homozygous for a dn mutant form of Ikaros lack all lymphocytes (Georgopoulos et al. 1994). The more severe phenotype of the $\mathrm{dn}$ Ik mutant suggested the presence of Ikaros homologs, leading to the identification of the related factors Aiolos (Morgan et al. 1997), Helios (Hahm et al. 1998, Kelley et al. 1998), and Eos (Cortes et al. 1999). In addition to its role in differentiation, Ikaros plays an important role in regulating cell proliferation. $\mathrm{Ik}+/-$ mice exhibit hyperactive T-cell receptor-mediated proliferative responses and eventually develop leukemias and lymphomas (Winandy et al. 1995, Avitahl et al. 1999). Animals heterozygous for the dn Ik-6 that lacks the DNA-binding domain develop T-cell lymphoproliferative disorders similar to human T-lymphoblastic leukemia or lymphoma, presumably as a result of inactivation of the normal allele (Winandy et al. 1995). Thus, Ikaros appears to be an essential regulator of early lymphocyte differentiation as well as differentiated cell expansion.

\section{The essential role of Ikaros in anterior pituitary cell growth and survival}

\section{Ikaros directs anterior pituitary cell expansion}

Our studies focused on detailed characterization of the striking neuroendocrine phenotypes of the Ikarosdeficient mouse model. In particular, the homozygous null mice exhibit a profound dwarf phenotype with nearly $95 \%$ mortality by 6 weeks of age (Ezzat et al. 2005a). The heterozygous Ik $+/-$ mice survive for 4-5 months and subsequently develop lymphoproliferative disorders with leukemic or atypical lymphoid infiltrates of several tissues. 
Although these animals were thought to develop infection with sepsis, complete autopsy examinations of our mice have not identified evidence of infection or sepsis. Rather, they show wasting with features of a profound metabolic/endocrine syndrome. Tissue and blood cultures were found to be negative. These animals have normal myeloid marrow elements, and there is no evidence of a neutrophilic response to infection. Moreover, reconstitution of Ikaros-deficient animals after birth with wild-type marrow demonstrated no measurable impact on size and overall health attributable to successful lymphocyte repopulation, supporting the hypothesis of an endocrine/metabolic basis for the phenotype (Ezzat et al. 2005a). These experiments provided compelling evidence for a lymphocyte-independent role for Ikaros in governing neuroendocrine system development.

\section{Ikaros modulates pituitary cell survival}

Animals heterozygous for dn forms of Ikaros (isoforms that lack the DNA-binding domain) develop T-cell disorders similar to human T-lymphoblastic leukemia or lymphoma, presumably by inactivation of the normal Ikaros allele (Winandy et al. 1995). Over-expression of a $\mathrm{dn}$ non-DNA-binding isoform Ik6 has been identified in a third of cases of B-cell acute leukemias (Nakase et al. 2000). Ikaros mutant mice display a decrease in expression of flk-2 and c-kit receptors, which may in part contribute to the early lymphopoietic phenotypes manifested in the absence of Ikaros (Nichogiannopoulou et al. 1999).

Having identified that Ikaros is expressed in the normal pituitary, we then examined its expression in neoplastic tissues. We detected Ik1 and Ik2/3 protein isoforms in human and mouse pituitary nuclear fractions (Ezzat et al. 2003). We also identified Ik6 expression in nearly $50 \%$ of human pituitary adenomas (Ezzat et al. 2003). Forced expression of this dn form of Ikaros resulted in histone 3 acetylation with the activation of the Bcl-XL promoter (Ezzat et al. 2006b). Parallel induction of the endogenous gene resulted in enhanced pituitary cell survival and evasion from apoptotic signals.

\section{Ikaros promotes anterior pituitary cell differentiation through cellular lipids}

To further determine the mechanisms of action of Ikaros in pituitary cell growth and differentiation, we used a cDNA microarray to uncover mediators of cholesterol uptake including the low-density lipoprotein receptor (LDL-R) and sterol regulatory element-binding protein 2 as targets of Ik action (Loeper et al. 2008). We showed that Ikaros regulates the LDL-R to alter metabolism in pituitary corticotroph cells. The DNA-binding Ikaros isoform, Ik1, binds and enhances activity of the LDL-R promoter. Ik1 decreases methylation and increases acetylation of histone 3 lysine 9 at the LDL-R promoter (Loeper et al. 2008). Confocal microscopy and quantitative fluorometry demonstrated enhanced LDL endocytosis in Ik1-transfected cells that exhibited abundant endoplasmic reticulum, large Golgi complexes, and prominent secretory granule formation, consistent with more robust cholesterol incorporation into functionally relevant membrane-rich organelles. Consistent with these data, LDL-R $-/-$ mice, like Ik $-/-$ mice, have decreased circulating levels of adrenocorticotropic hormone (Loeper et al. 2008). These findings expand the repertoire of Ikaros actions to include regulation of the cholesterol uptake metabolic pathway. This novel link between tumor suppression and differentiation provides a relationship between cellular metabolism and cancer, and has therapeutic implications for lipid-modifying drugs in Ikaros-associated disorders.

\section{Ikaros drives corticomelanotroph population expansion}

To quantify the in vivo effects of Ikaros on hypothalamicpituitary development, we examined in detail the effects of disruption of Ikaros on hypothalamic-pituitary architecture, cell number and area by quantitative morphometric analyses, proliferation rates, and apoptotic indices. Morphologic and morphometric examination of these animals reveals pituitary hypoplasia and dysgenesis in Ik-null mice. During fetal development, there is delayed progression of pituitary development with small, but architecturally normal pituitaries compared with wildtype littermates that have normal pituitary development (Shewchuk et al. 1999). After birth, the Ik-null mice continue to exhibit a marked reduction in pituitary mass. They have normal cytodifferentiation with the presence of GH-positive somatotrophs, PRL-positive lactotrophs, adrenocorticotrophin (ACTH)-positive corticotrophs, and thyrotrophin (TSH)-reactive thyrotrophs and gonadotrophs containing follicle-stimulating hormone and luteinizing hormone. However, there are marked reductions in the number of adenohypophysial cells, most strikingly involving the ACTH- and GH-producing populations. The pituitaries of homozygote mice exhibited a striking reduction of melanocorticotropes, which was most marked in the intermediate lobe. The heterozygote and homozygote animals showed no evidence of the classical expansion of the melanocorticotropes that normally proliferate to occupy the entire intermediate lobe.

To specifically examine the functional consequences of disrupted Ikaros signaling on pituitary corticomelanotroph development, we compared the hormonal and developmental profiles of Ik-null mice with their 
heterozygote and wild-type littermates (Ezzat et al. 2005a). Homozygote Ik-null mice demonstrated the lowest levels of ACTH in the systemic circulation. Levels of the POMC-derived melanocyte-stimulating hormone were also reduced in heterozygote but more strikingly in Ik-null mice. The functional consequences of a diminished population of POMC-producing corticomelanotroph cells were most evident in homozygote mice. Consistent with the trophic functions of ACTH on adrenocortical development and function, Ik-null mice display reduced circulating corticosterone levels compared with heterozygote and wild-type littermates. These changes were also reflected by the reduction of adrenal gland size with striking loss of adrenocortical mass, especially in homozygous Ik-deficient mice.

To examine the possibility that the diminished POMC production and adrenocortical insufficiency in Ikarosdeficient animals may be mediated through altered cytokine production by a dysregulated lymphocyte population, we examined the impact of wild-type bone marrow reconstitution on the endocrine phenotype. Five weeks after adoptive bone marrow transfer in neonatal mice, the proportion of splenic $\mathrm{CD} 4^{+}$and $\mathrm{CD} 8^{+} \mathrm{T}$-cells in the homozygous Ik-null recipients of Ik wild-type syngenic bone marrow was significantly restored compared with vehicle-treated controls. Despite successful reconstitution with normal lymphocytes, the phenotype of homozygote Ik-null animals was unchanged with persistently small pituitaries and reduction in POMC expression that was indistinguishable from vehicletreated age-matched homozygote animals. Moreover, neither heterozygote nor homozyogote Ik-deficient animals demonstrated a significant change in serum corticosterone levels following reconstitution with wildtype lymphocytes. These data provide evidence for a direct role of Ikaros in pituitary corticomelanotroph development and function independent of its influence on lymphopoiesis.

Recognizing that Ikaros mutant mice display adrenocortical insufficiency, we examined the impact of systemic hormone replacement on growth and survival of the Ik-null mouse. Glucocorticoid hormone treatment compared with vehicle alone resulted in significant weight increase in Ik-null mice; the effect was also present, but less evident, in heterozygote animals. By comparison, the same treatment displayed no significant impact on the growth of wild-type animals.

Overall, homozygote Ik-null mice display diminished life expectancy with nearly $5 \%$ mortality at birth, $40 \%$ by 3 weeks of age, and $95 \%$ by 6 weeks of age. Given the recognized functions of Ikaros on immune cell development and function, we performed complete organ surveys in search of opportunistic infections or malignancy. However, we found no evidence of either of these conditions to explain the observed mortality at these stages of development. Moreover, hormone treatment with glucocorticoids resulted in improved survival. In particular, Ik-null mice treated with the adrenocortical hormone demonstrated $100 \%$ survival beyond 6 weeks of age. For comparison, none of the vehicle-treated homozygote mice survived beyond the same time point.

\section{Ikaros binds and activates the POMC gene}

To determine the mechanism of Ikaros action responsible for the corticotroph insufficient phenotype, we analyzed the rat POMC promoter at five potential Ik-binding sites within the $-543 \mathrm{bp}$ promoter (Ezzat et al. 2005a). The importance of these putative Ikaros-binding sites was examined by electromobility shift assay and luciferase reporter assays. Two of the three sites that formed specific super-shifted complexes (fragment I $(-451 /-420)$ and fragment II $(-163 /-137))$ were identified to be functionally significant as determined through mutational analyses and co-transfection studies (Ezzat et al. 2005a). These Ikaros-binding sites in the POMC promoter are within close proximity ( $\sim 70 \mathrm{bp})$ upstream and downstream of the recently identified Tpit/Pitx1 regulatory element (Lamolet et al. 2001). The T-box factor Tpit is restricted to POMC-expressing corticomelanotroph pituitary cells (Lamolet $e t$ al. 2001). It activates POMC transcription in cooperation with contiguously bound Pitx1 by recruiting the SRC/p160 co-activators (Maira et al. 2003). Given the critical role of Ikaros in nuclear dimerization, the potential for physical interaction between Ikaros and Tpit needs to be specifically examined. Nevertheless, forced Ikaros expression, but not (dn) Ik6, results in induction of the endogenous POMC gene at the mRNA and protein levels (Ezzat et al. 2005a).

\section{Hypothalamic Ikaros actions}

\section{Ikaros directs somatotroph expansion through hypo- thalamic GHRH regulation}

Ikaros-deficient animals display a dwarf phenotype with body weight reaching only $\sim 50 \%$ of their wild-type littermates. This dwarfism is associated with diminished GH secretion as evidenced by a reduction of the GH-target growth factor insulin-like growth factor-I (IGF-I) by $>50 \%$ in homozygote animals. The Ikaros heterozygote-deficient animals display near normal somatic growth with more modest $(\sim 12 \%)$ reductions in IGF-I levels compared with wild-type mice. Healthy homozygote mice had the same body proportions as those of heterozygote and wild-type mice. At autopsy, internal organs were proportionally equivalent across the genotypes with the exception of the contracted pituitary and adrenal glands of homozygote mice. These features of GH deficiency are not a result of nonspecific 
generalized pituitary dysgenesis, since the animals have normal or slightly elevated circulating levels of TSH and PRL. Although these animals exhibit glucocorticoid deficiency that could account for GH insufficiency, glucocorticoid replacement that significantly improved viability resulted in only a minimal increase in body weight (Ezzat et al. 2005a).

The changes of proportionate postnatal dwarfism are indicative of GH deficiency in homozygous Ik-null mice. Again, to exclude the possibility of a secondary response to immunodeficiency, we performed bone marrow reconstitution studies and showed that hematopoietic replacement does not alter IGF-I levels and restore normal growth. We carried out administration of exogenous GH with a response of circulating IGF-I levels and body weight reflecting somatic growth (Ezzat et al. 2006a).

The pituitary somatotroph population displayed quantitative differences across the Ikaros genotypes during early postnatal development. Somatotrophs were present in approximately the same proportion of cells in all genotypes, but the glands are smaller in heterozygotes than in wild-type mice and are even smaller in homozygotes. The intensity of GH reactivity was also reduced in homozygote mice. GH positivity persists in the intermediate lobe of the lateral wings likely attributable to the lack of melanocorticotrope expansion that normally replaces intermediate lobe somatotrophs during development. Other cell types were not measurably affected; the number and area of immunoreactive thyrotrophs and gonadotrophs were not disproportionately different from those of wild-type littermates (Ezzat et al. 2006a).

To examine the regulation of GH by Ikaros, we examined pituitary mammosomatotroph GH4 cells that express abundant Ikaros. We found that Ikaros reciprocally regulates the GH and PRL genes (Ezzat et al. 2005b). In contrast to the expected results, we documented that wild-type Ikaros (Ik1) inhibits GH mRNA and protein expression while stimulating PRL mRNA and protein levels. Ikaros does not bind directly to the proximal $(-360)$ GH promoter. Instead, Ikaros significantly abrogates the effect of the histone deacetylation inhibitor trichostatin A on this promoter. Ikaros selectively deacetylates histone 3 residues on the proximal transfected or endogenous GH promoter and limits access of the Pit1 activator. By contrast, Ikaros acetylates histone 3 on the proximal PRL promoter and facilitates Pit1 binding to this region in the same cells (Ezzat et al. 2005b). These data provide evidence for Ikaros-mediated histone acetylation and chromatin remodeling in the selective regulation of pituitary $\mathrm{GH}$ and PRL hormone gene expression in mammosomatotroph cells.

This paradoxical result did not explain the dwarf phenotype of the Ik-null mice, and therefore another explanation for GH deficiency was sought in the hypothalamus. Abundant levels of Ikaros expression were identified in the hypothalamus of wild-type littermates. This staining was localized predominantly within the ventral hypothalamus and was colocalized with GHRH in hypothalamic neurons (Ezzat et al. 2006a). The number of positive cells reaches a maximum at embryonic day (e) 18 and shows a reduction in newborn mice; only scattered cells are positive in the adult brain. Ik-null mice showed a striking lack of hypothalamic GHRH. Over-expression of Ikaros enhanced GHRH promoter activity and induced endogenous GHRH gene expression, proving that there is a critical role of Ikaros in the functional regulation of GHRH (Ezzat et al. 2006a). These data unmask a wider role for Ikaros in the neuroendocrine system, highlighting a critical contribution to the development of the hypothalamic-pituitary somatotrophic axis.

\section{Higher central nevous system Ikaros actions}

Ikaros transcription factors are also expressed in the brain with predominance in the developing striatum where the putative binding site $\left(5^{\prime}\right.$-GGGA- $\left.3^{\prime}\right)$ in the promotor of the mouse and rat enkephalin genes is readily identified (Dobi et al. 1997). One study focusing on the relevance of Ik to the enkephalinergic system (Agoston et al. 2007) concluded that Ik is co-expressed with enkephalin mRNA and may act as a positive regulator of enkephalinergic specification in the developing striatum. While enkephalin is one of several striatal co-transmitters, expressed by a subset of medium spiny neurons (MSNs), there are many others, including gamma-aminobutyric acid (GABA), substance $\mathrm{P}$, and dopamine $\mathrm{D} 1$ and $\mathrm{D} 2$ receptors (reviewed in (Holt et al. 1997)). We found Ik in precursor cells of several neurostriatal compartments, suggesting that it may play a role as a general maturation factor for MSNs.

With these neuro-localization studies in mind, we proceeded to systematically examine the impact of loss of Ikaros on neurostriatal-mediated functions. We performed a battery of standardized neurobehavioral tests including the elevated plus maze (a measure of anxiety-like behavior), the acoustic startle response and pre-pulse inhibition tests (measures of motor and autonomic reaction), the pinch test (a measure of catalepsy), and contextual fear conditioning (measures of learning and emotion). None of these behavioral functions were significantly altered in Ik-null mice, however, it was specifically in the Porsolt's forced swim test (a measure of depression-like behavior), where we found $\mathrm{Ik}-/-$ mice spending significantly less time in immobility than their $\mathrm{Ik}+/+$ littermates (Kiehl et al. 2008). This phenotype is consistent with 


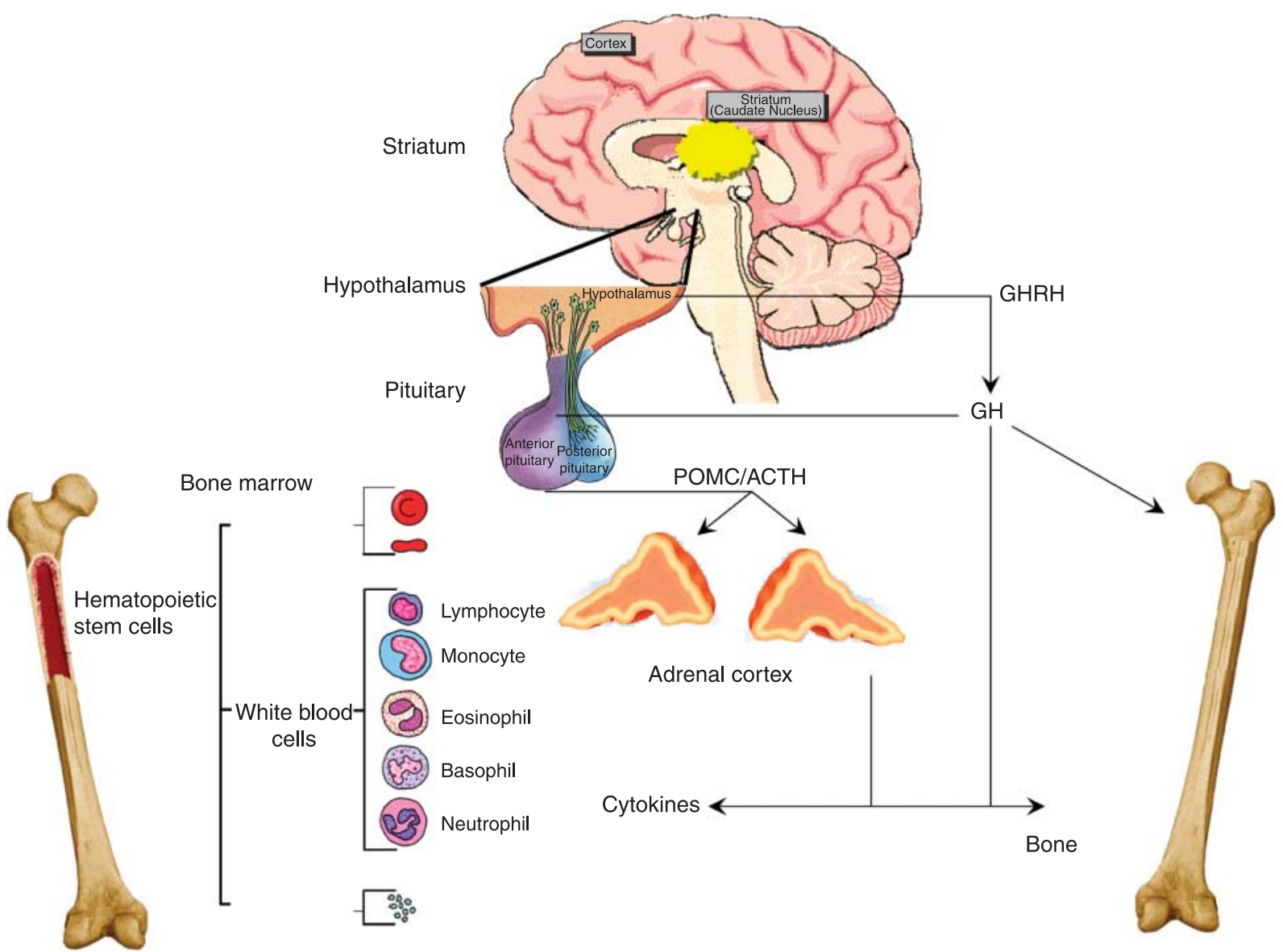

Figure 2 Ikaros is expressed in restricted sites throughout the neuroendocrine and hematopoietic systems. In the brain, the highest expression is in the medium spiny neurons of the striatum where the loss of function results in neurobehavioral changes characterized by an anti-depressant phenotype. In the hypothalamus, the median eminence of GHRH-containing neurons co-localize with Ikaros expression. Loss of Ikaros severely diminishes GHRH production and consequently GH and IGF-I activation. In the anterior pituitary, Ikaros is expressed in POMC-producing corticotrophs that govern the ACTH/adrenocortical axis. Ikaros is also expressed in the somatotrophs where it plays a direct inhibitory role. In the hematopoietic system, Ikaros' peak expression is in stem cells where it directs lymphoid lineage commitment. The multidimensional actions of Ikaros serve to sort and integrate diverse signals to regulate neuroendocrine-immune interactions through direct and indirect mechanisms.

reduced behavioral despair. These findings suggest that Ikaros-mediated neuro striatal cytodifferentiative functions impose significant and selective impact on depressive behavior (Kiehl et al. 2008). The neurochemical Ikaros targets involved in mediating these functions will undoubtedly provide novel pharmacologic opportunities for the treatment of psychoaffective disorders.

\section{Conclusions}

The identification of Ikaros as a transcriptional regulator of the POMC gene and consequently the ACTH-adrenal axis, its epigenetic control of the GH/PRL axis, and the exciting new finding of Ikaros in selected hypothalamic and striatal neurons, raises new questions about the pleiotropic neuroendocrine functions of this zinc finger protein (Fig. 2). The established functions of Ikaros as a mediator of early and late hematopoietic cell differentiation and proliferation provide further insights into the role of Ikaros as an integrator of endocrine, immune, and neurobehavioral functions. The studies reviewed here indicate that Ikaros can influence hypothalamic-pituitary cell development, differentiation, proliferation, and/or transformation, and provide evidence that these effects are mediated through overlapping and redundant cellular and nuclear targets. The balance between Ikaros and its $\mathrm{dn}$ Ik6 isoform appears to regulate multiple promoters through histone acetylation and methylationdependent mechanisms. The net influence of these interactions is ultimately expected to favor a chromatin environment that permits recruitment of an activating or repressing complex essential for gene-specific control. 


\section{Declaration of interest}

The authors have nothing to disclose. The authors declare that there is no conflict of interest that would prejudice the impartiality of this scientific work.

\section{References}

Agoston DV, Szemes M, Dobi A, Palkovits M, Georgopoulos K, Gyorgy A \& Ring MA 2007 Ikaros is expressed in developing striatal neurons and involved in enkephalinergic differentiation. Journal of Neurochemistry 102 1805-1816.

Asa SL \& Ezzat S 1998 The cytogenesis and pathogenesis of pituitary adenomas. Endocrine Reviews 19 798-827.

Asa SL \& Ezzat S 1999 Molecular determinants of pituitary cytodifferentiation. Pituitary 1 159-168.

Asa SL \& Ezzat S 2002 The pathogenesis of pituitary tumours. Nature Reviews. Cancer 2 836-849.

Avitahl N, Winandy S, Friedrich C, Jones B, Ge Y \& Georgopoulos K 1999 Ikaros sets thresholds for T cell activation and regulates chromosome propagation. Immunity 10 333-343.

Cortes M, Wong E, Koipally J \& Georgopoulos K 1999 Control of lymphocyte development by the Ikaros gene family. Current Opinion in Immunology 11 167-171.

Dobi A, Palkovits M, Ring MA, Eitel A, Palkovits CG, Lim F \& Agoston DV 1997 Sample and probe: a novel approach for identifying development-specific cis-elements of the enkephalin gene. Brain Research. Molecular Brain Research 52 98-111.

Ezzat S, Yu S \& Asa SL 2003 Ikaros isoforms in human pituitary tumors: distinct localization, histone acetylation, and activation of the $5^{\prime}$ fibroblast growth factor receptor-4 promoter. American Journal of Pathology 163 1177-1184.

Ezzat S, Mader R, Yu S, Ning T, Poussier P \& Asa SL 2005a Ikaros integrates endocrine and immune system development. Journal of Clinical Investigation 115 1021-1029.

Ezzat S, Yu S \& Asa SL $2005 b$ The zinc finger Ikaros transcription factor regulates pituitary growth hormone and prolactin gene expression through distinct effects on chromatin accessibility. Molecular Endocrinology 19 1004-1011.

Ezzat S, Mader R, Fischer S, Yu S, Ackerley C \& Asa SL 2006 $a$ An essential role for the hematopoietic transcription factor Ikaros in hypothalamic-pituitary-mediated somatic growth. PNAS 103 2214-2219.

Ezzat S, Zhu X, Loeper S, Fischer S \& Asa SL $2006 b$ Tumor-derived Ikaros 6 acetylates the Bcl-XL promoter to up-regulate a survival signal in pituitary cells. Molecular Endocrinology 20 2976-2986.

Georgopoulos K, Moore DD \& Derfler B 1992 Ikaros, an early lymphoid-specific transcription factor and a putative mediator for T cell commitment. Science 258 808-812.

Georgopoulos K, Bigby M, Wang JH, Molnar A, Wu P, Winandy S \& Sharpe A 1994 The Ikaros gene is required for the development of all lymphoid lineages. Cell 79 143-156.

Georgopoulos K, Winandy S \& Avitahl N 1997 The role of the Ikaros gene in lymphocyte development and homeostasis. Annual Review of Immunology 15 155-176.

Hahm K, Ernst P, Lo K, Kim GS, Turck C \& Smale ST 1994 The lymphoid transcription factor LyF-1 is encoded by specific, alternatively spliced mRNAs derived from the Ikaros gene. Molecular Cell Biology 14 7111-7123.

Hahm K, Cobb BS, McCarty AS, Brown KE, Klug CA, Lee R, Akashi K, Weissman IL, Fisher AG \& Smale ST 1998 Helios, a T cell-restricted Ikaros family member that quantitatively associates with Ikaros at centromeric heterochromatin. Genes and Development 12 782-796.
Holt DJ, Graybiel AM \& Saper CB 1997 Neurochemical architecture of the human striatum. Journal of Comparative Neurology 384 1-25.

Kelley CM, Ikeda T, Koipally J, Avitahl N, Wu L, Georgopoulos K \& Morgan BA 1998 Helios, a novel dimerization partner of Ikaros expressed in the earliest hematopoietic progenitors. Current Biology $8508-515$.

Kiehl TR, Fischer SE, Ezzat S \& Asa SL 2008 Mice lacking the transcription factor Ikaros display behavioral alterations of an antidepressive phenotype. Experimental Neurology 211 107-114.

Lamolet B, Pulichino AM, Lamonerie T, Gauthier Y, Brue T, Enjalbert A \& Drouin J 2001 A pituitary cell-restricted T box factor, Tpit, activates POMC transcription in cooperation with Pitx homeoproteins. Cell $104849-859$

Loeper S, Asa SL \& Ezzat S 2008 Ikaros modulates cholesterol uptake: a link between tumor suppression and differentiation. Cancer Research 68 3715-3723.

Maira M, Couture C, Le Martelot G, Pulichino AM, Bilodeau S \& Drouin J 2003 The T-box factor Tpit recruits SRC/p160 co-activators and mediates hormone action. Journal of Biological Chemistry 278 46523-46532.

Molnar A \& Georgopoulos K 1994 The Ikaros gene encodes a family of functionally diverse zinc finger DNA-binding proteins. Molecular Cell Biology 14 8292-8303.

Molnar A, Wu P, Largespada DA, Vortkamp A, Scherer S, Copeland NG, Jenkins NA, Bruns G \& Georgopoulos K 1996 The Ikaros gene encodes a family of lymphocyte-restricted zinc finger DNA binding proteins, highly conserved in human and mouse. Journal of Immunology 156 585-592.

Morgan B, Sun L, Avitahl N, Andrikopoulos K, Ikeda T, Gonzales E, Wu P, Neben S \& Georgopoulos K 1997 Aiolos, a lymphoid restricted transcription factor that interacts with Ikaros to regulate lymphocyte differentiation. EMBO Journal 16 2004-2013.

Nakase K, Ishimaru F, Avitahl N, Dansako H, Matsuo K, Fujii K, Sezaki N, Nakayama H, Yano T, Fukuda S et al. 2000 Dominant negative isoform of the Ikaros gene in patients with adult B-cell acute lymphoblastic leukemia. Cancer Research 60 4062-4065.

Nichogiannopoulou A, Trevisan M, Neben S, Friedrich C \& Georgopoulos K 1999 Defects in hemopoietic stem cell activity in Ikaros mutant mice. Journal of Experimental Medicine 190 1201-1214.

Shewchuk BM, Asa SL, Cooke NE \& Liebhaber SA 1999 Pit-1 binding sites at the somatotrope-specific DNase I hypersensitive sites I, II of the human growth hormone locus control region are essential for in vivo hGH-N gene activation. Journal of Biological Chemistry 274 35725-35733.

Sun L, Liu A \& Georgopoulos K 1996 Zinc finger-mediated protein interactions modulate Ikaros activity, a molecular control of lymphocyte development. EMBO Journal 15 5358-5369.

Wang JH, Nichogiannopoulou A, Wu L, Sun L, Sharpe AH, Bigby M \& Georgopoulos K 1996 Selective defects in the development of the fetal and adult lymphoid system in mice with an Ikaros null mutation. Immunity 5 537-549.

Winandy S, Wu P \& Georgopoulos K 1995 A dominant mutation in the Ikaros gene leads to rapid development of leukemia and lymphoma. Cell 83 289-299.

Winandy S, Wu L, Wang JH \& Georgopoulos K 1999 Pre-T cell receptor (TCR) and TCR-controlled checkpoints in T cell differentiation are set by Ikaros. Journal of Experimental Medicine 190 1039-1048.

Received in final form 6 May 2008

Accepted 29 May 2008

Made available online as an Accepted Preprint 29 May 2008 